\title{
Role of Ultrasonography in Thyroid Nodules with Pathological Correlation
}

\author{
Subhash C. Sylonia ${ }^{\circledR 1}$, Pranay Kumar ${ }^{\circledR 2}$ \\ ${ }^{1}$ Professor \& Head, Department of Radiodiagnosis \& Imaging, Saraswathi Institute of Medical Sciences, Hapur, Uttar Pradesh, India, ${ }^{2}$ Post Graduate Resident, \\ Department of Radiodiagnosis \& Imaging, Saraswathi Institute of Medical Sciences, Hapur, Uttar Pradesh, India.
}

\section{Abstract}

Background: Thyroid nodule imaging had been a challenging task. Ultrasound has been the modality of choice for detection \& evaluation of these lesions. This study was done to identify the accuracy of various imaging features in thyroid nodules that are associated with benignity and malignancy and the overall accuracy of ultrasound in determining malignant nodules. The objective of the study are: (1) Ultrasonographic evaluation of thyroid to characterize the thyroid disease and nodules (benign vs malignant) and their morphologic evaluation. (2) Sensitivity and Specificity on the basis of radiological, and cytopathological correlation for suspected thyroid lesions. Subjects and Methods: A total of seventy patients (70) of both sexes and different age group, meeting the inclusion criterion, were the part of present prospective study. The cases referred to the Department of Radio-diagnosis and imaging from OPD/IPD of Saraswathi Institute of Medical sciences, Hapur from October'2017 to July'2019. An informed consent was taken from all the patients subjected for evaluation. Results: The collective data was analysed for Ultrasound findings of thyroid nodule which presented clinically as solitary or multiple thyroid nodules. The total number of cases analysed was 70 out of which 48 were found to have solitary/multinodular lesions clincally. On ultrasound evaluation out of 48 patients 38 had solitary/multinodular lesions. Conclusion: High resolution sonography is recommended as the primary imaging modality in evaluation of thyroid diseases. It has a high sensitivity and specificity in the diagnosis of thyroid diseases.

Keywords: Ultrasonography, Thyroid Nodules.

Corresponding Author: Pranay Kumar, Post Graduate Resident, Department of Radiodiagnosis \& Imaging, Saraswathi Institute of Medical Sciences, Hapur, Uttar Pradesh, India.

E-mail: pranaybhu26@gmail.com

Received: 11 February 2020

Revised: 19 March 2020
Accepted: 31 March 2020

Published: 05 July 2020

\section{Introduction}

Thyroid nodules are extremely common, found at palpation in $4 \%$ to $7 \%$ of an asymptomatic population, ${ }^{[1,2]}$ in $17 \%$ to $27 \%$ of cases at USG, ${ }^{[3-5]}$ and in $50 \%$ of cases at autopsy. ${ }^{[6]}$ USG is the modality of choice in a clinically suspected case of thyroid lesion and the most sensitive imaging modality to detect a clinically non-palpable nodule of $2-3 \mathrm{~mm}$ size and allows a more accurate morphological characterization of the lesion including the size and the number of thyroid nodules, the volume of thyroid tissue and could well differentiate with extra-thyroid neck masses. An addition of color and spectral Doppler imaging that determines the vascular pattern of thyroid diseases has been found to be very useful tool in screening the thyroid nodule for malignancy. ${ }^{[7,8]}$

\section{Subjects and Methods}

This prospective study was conducted from 1st October 2017 to 30th July' 2019 on the cases referred from OPD/IPD of the SIMS Hospital, to the Department of Radio-diagnosis, SIMS, Hapur in collaboration with the Department of Pathology, Otolaryngology, and Surgery. Sonography was performed using Philips clear vue 350 USG unit with multi-frequency probe of 5.0 to $13 \mathrm{MHz}$ probe. A phased array curvilinear broadband transducer of 2 to $5 \mathrm{MHz}$ frequency was also used to assess larger lesions. Fine needle aspiration cytology (FNAC) and Histo-pathological correlation, if patient is subjected to surgery, in thyroid diseases was also done. Clinical findings or investigations and imaging findings were co - related. Distribution of cases according to post ultrasound diagnosis was done.

\section{Inclusion criteria}


- All the cases presented and referred on clinical grounds for suspicion of thyroid diseases with or without deranged thyroid function test.

- Patients who presented with a palpable thyroid nodule.

- Patients with deranged thyroid function tests.

- Incidentally discovered thyroid nodules.

\section{Exclusion criteria}

- All patients with previous FNAC or biopsy proven diagnosis.

- Patients who had previously undergone thyroid surgery for any reason.

\section{Results}

In the present study, there was a female preponderance and out of the 70 patients $61 / 70(87.4 \%)$ werefemales and $9 / 70$ $(12.6 \%)$ were males. The M:F ratio in the study group is 1 : 6.7 showed a female preponderance for thyroid diseases. The study group composed of patients ranging from 7 to 92 years of age. Majority of the patients were in the age group of 3140 years, accounting for $31.5 \%$ of the cases. The mean age in the study was33.6years. Only one patient above 70 years of age was included in the study. Female preponderance was noted in patients with thyroid disorders in all age groups except between 61 to 70 years, where there was equal ratio of males and females. Five patients with clinical suspicion of thyroid disease, were found normal at sonography.

Out of 48/70 (68.57\%) patients presented with solitary thyroid nodule on clinical grounds and out of these 17/48 (35.4\%) of patients diagnosed as Solitary Thyroid Nodule (STN) clinically were found to have a multi-nodular (MNG) thyroid disease at high resolution USG.21/48(43.75\%) patients were found to have sonographic STN while 10/48(20.85\%) of these patients had absence of nodules on ultrasound.

Most of the nodules of colloid goiter were isoechoic $16 / 21(76.4 \%)$, followed by heteroechogenecity in $14.2 \%$. Consistency of 12/21 (57\%) nodules was mixed solid- cystic, followed by solid nodules in $28.5 \%$. Only $14.2 \%$ of cases were purely cystic in consistency.

Calcification was seen in $9(42.7 \%)$ cases out of 21 nodules, among which $28.5 \%$ were coarse calcifications and $14.2 \%$ were comet tail type of calcification. A thin well defined, complete halo was seen in $85.7 \%$ of these lesions. Incomplete halo was seen $14.3 \%$ of cases. Most of the nodules (15/21) showed a predominantly peripheral flow pattern $(71.4 \%)$. The $23.8 \%$ of the nodules showed both peripheral and intranodular vascularity. Only one nodule howed absence of vascularity.

17 out of 70 patients were diagnosed as multinodulargoitre (MNG) on ultrasonography and were subsequently proved on FNAC. $70.5 \%$ of the MNG were solid- cystic in consistency, followed by purely solid consistency in $23.5 \%$ and purely cystic in $6 \%$ cases. $52.9 \%$ of cases were isoechoic in echopattern, followed by heteroechoicechopattern in 35.5\%. Anechoic and hyperechogenecity were seen in $5.8 \%$ of cases (1) each. Calcifications were present in $29.4 \%$ (5) cases and all were coarse type. Thin regular complete halo were present in $88.3 \%$ (15) cases. Halo was absent in $11.7 \%$ (2) cases. On CDI, internodular vascularity was seen in $82.6 \%$ cases followed by intranodular flow in $5.8 \%$ cases. No vascularity (absent flow) was seen in $11.6 \%$ cases.

Two patients of Grave's disease had solid, enlarged, bulky, diffusely hypoechoic thyroid with few echogenic strands. The patients exhibited the thyroid inferno pattern at colour flow imaging.

$89.4 \%$ of the cases presented with hypoechoicechotexture and $10.6 \%$ cases presented with heteroechoicechotexture. There was nodularity in two of the patients in the form of discrete nodules in a background of diffusely altered parenchyma.

Two cases of papillary neoplasm showed heteroechoic thyroid parenchyma with ill defined multiple nodules with solid-cystic changes and cystic degeneration (Image no. 20) with absence of halo. Both cases showed presence of microcalcifications (Image no. 22,23). Extrathyroid involvement in the form of lymph nodes was seen in both cases.

Since the following diseases were more commonly encountered in our study, we did a comparison of their various ultrasonographic features.

Table 1: Comparison of USGCharacterstics of Various Diseases

STN MNG Thyroiditi Malignancy

$\begin{array}{lllll}\text { Total Cases } & 21 & 17 & 19 & 2\end{array}$

STN (Solitary Thyroid Nodule); MNG (Multi Nodular Goitre)

Table 2: Comparative Chart of Consistency of Various Diseases of Thyroid On USG

\begin{tabular}{|l|llll}
\hline Consistency & STN & MNG & Thyroiditis & Malignancy \\
\hline Solid & 6 & 4 & 19 & 0 \\
\hline Solid- Cystic & 12 & 12 & 0 & 2 \\
\hline Cystic & 3 & 1 & 0 & 0 \\
\hline Total & 21 & 17 & 19 & 2 \\
\hline
\end{tabular}

STN (Solitary Thyroid Nodule); MNG (Multi Nodular Goitre)

Coarse calcifications are seen predominantly in the benign nodules in Solitary or multi nodular goitre. Comet tail calcifications are seen mainly in solitary thyroid nodules. Microcalcifications are predominantly seen in malignant lesions, however an also be seen in nodular goitre.

Thick incomplete irregular halo or absent halo is seen in malignant lesions. 
Table 3: Comparative Chart of Echopattern of Various Diseases of Thyroid on USG

\begin{tabular}{lllll}
\hline Echopattern & STN & MNG & Thyroidit & Malignancy \\
Hypoechoic & 0 & 0 & 17 & 0 \\
Isoechoic & 16 & 9 & 0 & 0 \\
Heteroechoic & 3 & 6 & 2 & 2 \\
Hyperechoic & 1 & 1 & 0 & 0 \\
Anechoic & 1 & 1 & 0 & 0 \\
Total & 21 & 17 & 19 & 2 \\
\hline
\end{tabular}

STN (Solitary Thyroid Nodule); MNG (Multi Nodular Goitre)

Table 4: Comparative Study of Calcification in Various Diseases on USG

\begin{tabular}{lllll}
\hline Calcification & STN & MNG & \multicolumn{2}{l}{ Thyroiditi } \\
Coarse & 6 & 5 & 0 & 1 \\
Microcalcificati & 0 & 0 & 0 & 2 \\
$\begin{array}{l}\text { Rim calcifica- } \\
\text { tion }\end{array}$ & 0 & 0 & 0 & 0 \\
Comet tail & 3 & 0 & 0 & 0 \\
\hline
\end{tabular}

STN (Solitary Thyroid Nodule); MNG (Multi Nodular Goitre)

\section{Table 5: Type of Halo in Various Diseases}

\begin{tabular}{llll}
\hline Halo & STN & MNG & Malignancy \\
Thin, regular complete & 18 & 15 & 0 \\
$\begin{array}{l}\text { Thick incomplete irregu- } \\
\text { lar }\end{array}$ & 3 & 0 & 0 \\
Absent & 0 & 2 & 2 \\
Total & 21 & 17 & 2 \\
\hline
\end{tabular}

STN (Solitary Thyroid Nodule); MNG (Multi Nodular Goitre)

Table 6: Type of Margins of Lesions in Various Diseases

\begin{tabular}{llll} 
Margins & STN & MNG & Malignany \\
Well & 21 & 17 & 0 \\
Defined & & & \\
Ill Defined & 0 & 0 & 2 \\
\hline
\end{tabular}

STN (Solitary Thyroid Nodule); MNG (Multi Nodular Goitre)

Well defined regular margins are seen in all the benign lesions. Ill-defined incomplete margins are seen in malignant lesions.

Diffuse increased vascularity is seen most frequently in thyroiditis and Graves' disease, less commonly it can also be seen in multinodulargoitre cases. High velocity flow pattern is seen in Graves disease and low and medium velocity flow is seen in thyroiditis cases. Intranodular and peripheral vascular flow are seen in solitary and multinodular thyroid nodules and in malignancy.
Table 7: Pattern of Vascularity in Various Diseases

\begin{tabular}{|c|c|c|c|c|c|}
\hline Vascularity & & STN & MNC & Thyrois & Malignancy \\
\hline Peripheral & & 15 & 14 & 0 & 0 \\
\hline Avascular & & 1 & 2 & 0 & 0 \\
\hline $\begin{array}{l}\text { Intranodular } \\
\text { peripheral }\end{array}$ & and & 5 & 1 & 0 & 2 \\
\hline Diffuse & & 0 & 0 & 19 & 0 \\
\hline Total & & 21 & 17 & 19 & 2 \\
\hline
\end{tabular}

STN (Solitary Thyroid Nodule); MNG (Multi Nodular Goitre) 


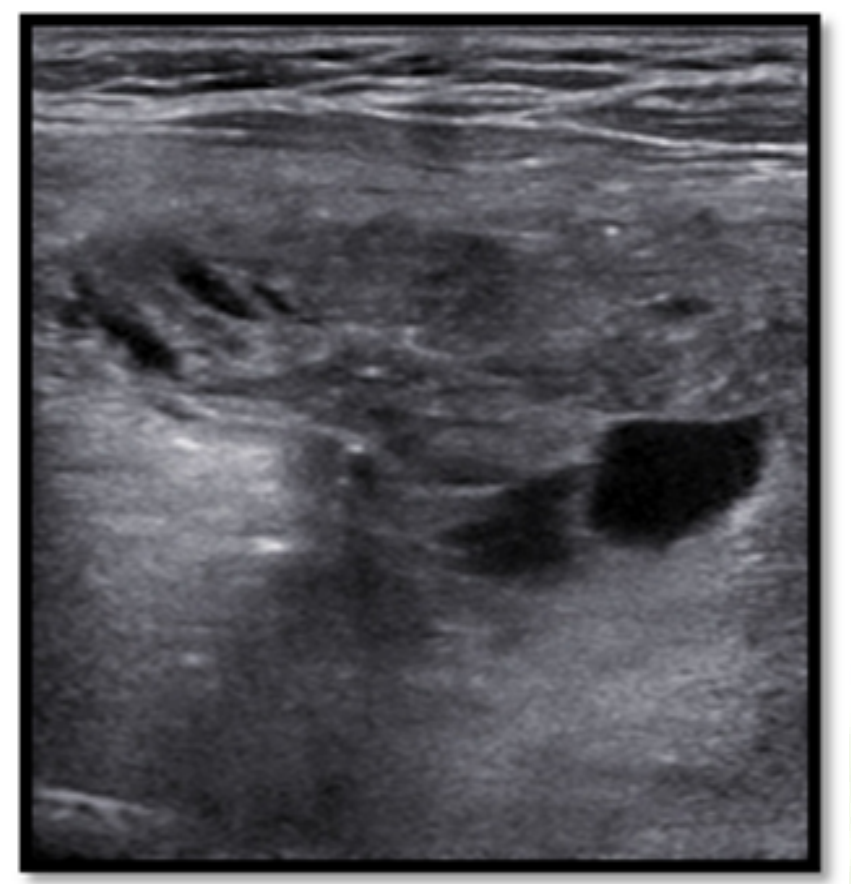

HRS USG in a 92 year old female showing relatively hypoechoic nodules with ill-defined margins in the left lobe of thyroid with absent halo and multiple microcalcifications. $\mathrm{CD}$ revealed predominant internal vascularity.

\section{Discussion}

The present study was undertaken to evaluate the role of High Resolution Ultra-Sonography in evaluation of thyroid diseases. The largest group of patients were in the 31-40 year age group with $87.4 \%$ of the patients were females and $12.6 \%$ were males. The overall sex ratio was $\mathrm{M}: \mathrm{F}=1: 6.7$. A similar female preponderance was noted by Solbiati et al, in 1985 and Nam Goong et al, in 2003 in their respective studies. ${ }^{\text {[9-12] }}$

Out of 48 patients with clinical suspicion of thyroid nodule, $35.4 \%$ of patients diagnosed as Solitary Thyroid Nodule clinically were found to have a multinodular thyroid disease at high resolution sonography. $21(43.75 \%)$ patients were sonographically proven to be STN. 10 patients who clinically suspected to be STN were diagnosed as other thyroid disorders and showed no evidence of nodularity on Ultrasonography. ${ }^{[13-15]}$

The commonest thyroid pathology encountered in the study was hyperplastic goiter $(55.7 \%)$. Of these majority were nontoxic $(54.2 \%)$. Toxic nodular goiter accounted for $1.4 \%$ of the cases. Solbiati et al, ${ }^{[16]}$ in 1992 reported hyperplasia of thyroid as the commonest thyroid pathology.

Of the 38 patients with nontoxic hyperplastic goiter, 21 patients had solitary thyroid nodule while 17 had multinodular goiter. In patients with STN, consistency of $57 \%$ nodules was mixed solid- cystic, followed by solid nodules in $28.5 \%$. Only $14.2 \%$ of STN cases were purely cystic in consistency. $70.5 \%$ of the MNG were solid- cystic in consistency, followed by purely solid consistency in $23.5 \%$ cases.

Majority of the patients with nontoxic nodular goiter showed isoechoic lesions with areas of cystic degeneration as were observed by Solbiati et al, ${ }^{[16]} 1992$. Coarse calcification was seen in $30.5 \%$ of cases of nontoxic hyperplastic goiter. A thin well defined and complete halo was seen in $85 \%$ of patients presenting as sonographic STN and $88 \%$ of multinodular goiter. Similar gray scale findings have been reported by Solbiati 1992. ${ }^{[16]}$ Doppler study in cases of USG STN revealed $71.4 \%$ of the nodules to have peripheral flow only while $23.8 \%$ of the nodules had both intranodular and peripheral flow.

Toxic multinodular goitre patients showed heteroechoic echopattern with high velocity internodular flow. Similar findings have been reported by Solbiati et al 1992. ${ }^{[16]}$

Both the patients of Graves' disease included in the study showed solid, bulky, diffusely hypoechoic thyroid parenchyma with prominent septae and capsule. The findings were concurrent with James 1985. ${ }^{[8]}$ Both patients exhibited the thyroid inferno pattern at colour flow imaging with similar findings noted by Clark et al 1995.

There were 19 cases of thyroiditis in the study. All the cases had solid thyroid parenchyma \& showed diffusely hypoechoic thyroid gland with echogenic septations with reactive cervical lymphadenopathy was seen in two of these patients with similar findings have been reported in thyroiditis by Solbiati et al 1992. ${ }^{[16]}$ Doppler study revealed diffuse parenchymal flow of low to medium velocity in all the patients consistent with findings of Clark et al 1995. [14]

There were two patients with papillary thyroid carcinoma in our study showed heteroechoic echopattern with a mixed consistency. There was multinodularity in both the cases and the nodules showed ill-defined margins. Solbiati et al, ${ }^{[16]}$ in 1985 showed that margin was ill defined and irregular in $69.7 \%$ and well-defined in $30.3 \%$. Thyroid lesion with welldefined margin suggests benign pathology. ${ }^{[8]}$ These findings were consistent with those in our study.

There was presence of coarse calcifications in one patient while both patients showed the presence of microcalcifications. In a study done by Mary C. Frates, Benson C.B. et al, it was seen that the presence of any calcification within nodule raises the likelihood of malignancy. ${ }^{[17]}$

Ill-defined incomplete margins were seen in both the cases of papillary carcinoma. Koike et al 2001, ${ }^{[6,8]}$ reported that $56.1 \%$ of all malignant nodules had irregular margins suggesting irregular margin as a common finding of malignancy in thyroid nodules. 
Presence of halo has been observed more often in benign lesions (Simeone et al 1982, Solbiati et al 1985). Similar observations were made in the present study which revealed $85.7 \%$ of the benign STN possessing a halo. Both the malignant lesions in the study showed presence of absent or irregular halo.

Thyroid calcifications can be classified as coarse calcification, peripheral or microcalcifications. Thyroid microcalcifications are psammoma bodies which are 10-100um round laminar calcific deposits. At USG, microcalcifications appear as punctate hyperechoic foci without acoustic shadowing. Microcalcifications are found in $29-59 \%$ of all thyroid carcinomas, ${ }^{[7,8]}$ most commonly in papillary thyroid carcinomas.

In the present study, both the cases of papillary thyroid carcinoma showed presence of microcalcification. In 2004, Iannucilli et al stated that presence of microcalcifications is the only statistically significant predictor of malignancy in thyroid nodules. ${ }^{[15]}$

Peripheral calcification is seen most commonly in multinodular goitre but may also be present in malignancy. ${ }^{[13]} 5.8 \%$ of MNG cases showed presence of peripheral calcification in the present study.

Invasion of anatomic structures around thyroid was observed in $1(50 \%)$ patient of malignancy. The patient showed extension of the lesion in the tracheoesophageal groove with infiltration into the trachea which was confirmed on CT. Local invasion into strap muscles, tracheal infiltration and carotid entrapment was seen in $26 \%$ of the thyroid malignancies in the study group of Hayashi et al 1986. ${ }^{[11]}$

Cervical adenopathy was seen in both cases of thyroid malignancy. One patient showed presence of microcalcification in the lymph nodes as well. Solbiati 1992 described microcalcification and cystic degeneration in the nodes as pathognomic for metastatic nodes from thyroid primary. ${ }^{[16]}$

Confirmation of thyroid tissue was done by FNAC and thyroid scintigraphy.

Results of our study show that high resolution sonography had sensitivity of $96.88 \%$ and specificity of $83.33 \%$ in diagnosing thyroid lesions with a positive predictive value of $98.41 \%$ and NPV of $71.43 \%$.

\section{Conclusion}

High resolution sonography is a useful modality for evaluation of thyroid diseases. It is reliable in distinguishing normal from abnormal thyroid. Thyroid sonography is useful in defining whether the patient has a diffuse abnormality, a multinodular pathology or a solitary nodule. It is an excellent modality for morphological characterization of thyroid lesions. High resolution sonography can differentiate benign from malignant thyroid nodules and masses in majority of cases. Sonography is very sensitive in detecting local invasion by thyroid malignancies. However, the substernal and retrosternal components of thyroid masses cannot be adequately imaged due to technical constraints. It is useful in diagnosis and follow up of diffuse thyroid diseases i.e. Grave's disease and thyroiditis. Sonography can easily and reliably exclude the possibility of ectopic thyroid in suspected cases of thyroglossal cysts or midline neck swellings. It is a useful imaging modality in evaluation of thyroid in children and pregnant women. Sonography can be used precisely to guide FNAC from impalpable thyroid lesions. Color flow imaging gives useful information about the vascular status of the lesions in thyroid and helps in characterizing indeterminate lesions in some cases.

\section{References}

1. Chaudhary V, Bano S. Imaging of the thyroid: Recent advances. Indian J Endocr Metab. 2012;16(3):371-371. Available from: https://dx.doi.org/10.4103/2230-8210.95674.

2. Desforges JF, Mazzaferri EL. Management of a Solitary Thyroid Nodule. N Engl J Med. 1993;328(8):553-559. Available from: https://dx.doi.org/10.1056/nejm199302253280807.

3. Carroll B. Asymptomatic thyroid nodules: incidental sonographic detection. AJR Am J Roentgenol. 1982;138:499-501.

4. Woestyn J, Afschrift M, Schelstraete K, Vermeulen A. Demonstration of nodules in the normal thyroid by echography. Brit J Radiol. 1985;58(696):1179-1182. Available from: https: //dx.doi.org/10.1259/0007-1285-58-696-1179.

5. Brander AEE, Viikinkoski VP, Nickels JI, Kivisaari LM. Importance of Thyroid Abnormalities Detected at US Screening: A 5-year Follow-up. Radiology. 2000;215(3):801806. Available from: https://dx.doi.org/10.1148/radiology.215. 3.r00jn07801.

6. Mortensn JD, Woolner LB, Bennett WA. Gross and microscopic findings in clinically normal thyroid glands. J Clin Endocrinol Metab. 1955;15(10):1270-1280. Available from: https://dx.doi.org/10.1210/jcem-15-10-1270.

7. Papini E, Guglielmi R, Bianchini A, Crescenzi A, Taccogna S, Nardi F, et al. Risk of Malignancy in Nonpalpable Thyroid Nodules: Predictive Value of Ultrasound and Color-Doppler Features. J Clin Endocrinol Metab. 2002;87(5):1941-1946. Available from: https://dx.doi.org/10.1210/jcem.87.5.8504.

8. Khizer AT, Raza S, Slehria AUR. Diffusion-weighted MR Imaging and ADC Mapping in Differentiating Benign from Malignant Thyroid Nodules. J Coll Physicians Surg Pak. 2015;25(11):785-788.

9. Solbiati L, Pra LD, Ierace T, Bellotti E, Derchi LE. Highresolution sonography of the recurrent laryngeal nerve: anatomic and pathologic considerations. Am J Roentgenol. 1985;145(5):989-993. Available from: https://dx.doi.org/10. 2214/ajr.145.5.989.

10. Chaudhary V, Bano S. Thyroid ultrasound. Indian J Endocrinol Metab. 2013;17(2):219-277. Available from: https://dx.doi. org/10.4103/2230-8210.109667. 
11. Hayashi N, Tamaki N, Yamamoto K, Senda M, Yonekura Y, Misaki T, et al. Real-Time Ultrasonography of Thyroid Nodules. Acta Radiol. 1986;27(4):403-408. Available from: https://dx.doi.org/10.1177/028418518602700406.

12. Nam-Goong IS, Kim HY, Gong G, Lee HK, Hong SJ, Kim $\mathrm{WB}$, et al. Ultrasonography-guided fine-needle aspiration of thyroid incidentaloma: correlation with pathological findings. Clin Endocrinol. 2004;60(1):21-28. Available from: https: //dx.doi.org/10.1046/j.1365-2265.2003.01912.x.

13. Kovacevic DO, Skurla MS. Sonographic diagnosis of thyroid nodules: correlation with the results of sonographically guided FNAB. J Clin Ultrasound. 2007;35(2):63-70. Available from: https://doi.org/10.1002/jcu.20287.

14. Clark KJ, Cronan JJ, Scola FH. Color Doppler sonography: Anatomic and physiologic assessment of the thyroid. J Clin Ultrasound. 1995;23(4):215-223. Available from: https://dx. doi.org/10.1002/jcu.1870230403.

15. Iannuccilli JD, Cronan JJ, Monchik JM. Risk for Malignancy of Thyroid Nodules as Assessed by Sonographic Criteria. J Med Ultrasound. 2004;23(11):1455-1464. Available from: https://dx.doi.org/10.7863/jum.2004.23.11.1455.

16. Jain N, Brahmbhatt P, Chaudhuri CR, Singhal A. Ultrasonography of Thyroid Lesions with Clinicopathological Correlation.
IJRR. 2020;7(1):142-158. Available from: IJRR.

17. Frates MC, Benson CB, Charboneau JW, Cibas ES, Clark OH, Coleman BG, et al. Management of thyroid nodules detected at US: society of Radiologists in Ultrasound consensus conference statement. Radiology. 2005;237(3):794-800. Available from: https://doi.org/10.1148/radiol.2373050220.

Copyright: (C) the author(s), 2020. It is an open-access article distributed under the terms of the Creative Commons Attribution License (CC BY 4.0), which permits authors to retain ownership of the copyright for their content, and allow anyone to download, reuse, reprint, modify, distribute and/or copy the content as long as the original authors and source are cited.

How to cite this article: Sylonia SC, Kumar P. Role of Ultrasonography in Thyroid Nodules with Pathological Correlation. Asian J. Med. Radiol. Res. 2020;8(1):100-105.

DOI: dx.doi.org/10.47009/ajmrr.2020.8.1.18

Source of Support: Nil, Conflict of Interest: None declared.

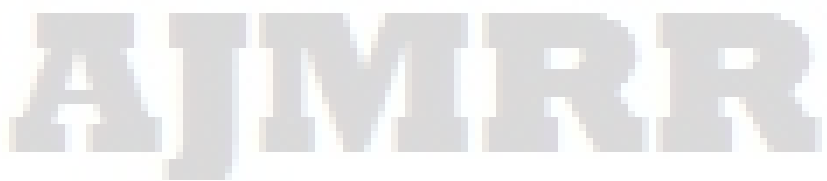

